\section{§21. Dynamics of Orientationally Ordered Domains in a Short Chain-molecule System: -Size Dependence of Domain Oscillation-}

Nakamura, H., Fujiwara, S., Sato, T.

As the fundamental process of structure formation for short chain molecules[1,2,3], the coalescence of two orientationally ordered domains were investigated by numerical simulation[4].

It was also demonstrated[4] that domains move collectively as if they were rigid bodies in spite of the non-bonded short-range interaction potential (Lennard-Jones potential) among chain molecules.

Dynamics of domain is investigated for several domain size (See Fig.1). Average angle $\theta$ for right or left domains is plotted for $(61+61)$ and $(61+29)$ chain-molecule system in Fig. 2.

From this figure, inertia of domain depends on its size. This property is not surprising, because the ordinal rigid body has this property. However, we find that the two cases in Fig.2. have the same periods of the domain's oscillation. This fact is not observed in motions of the ordinary rigid body.

\section{$(61,29)$}
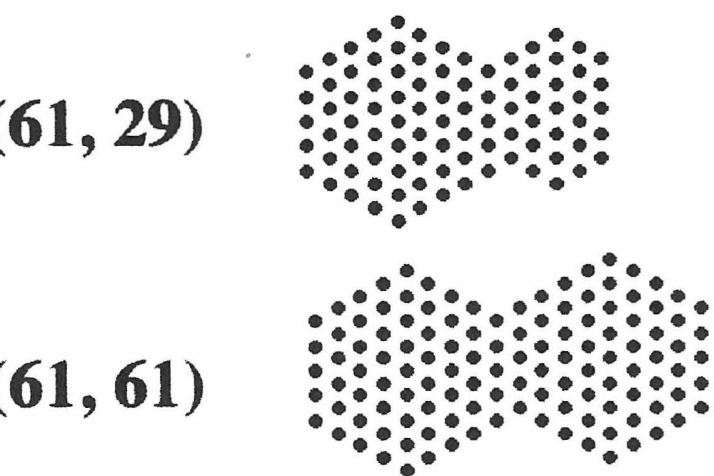

Fig. 1 Configuration of short chain molecules for two cases. The upper one is $(61+29)$ chain-molecule system and the lower is $(61+61)$ chain-molecule system. (a) (61+29) Chain Molecules

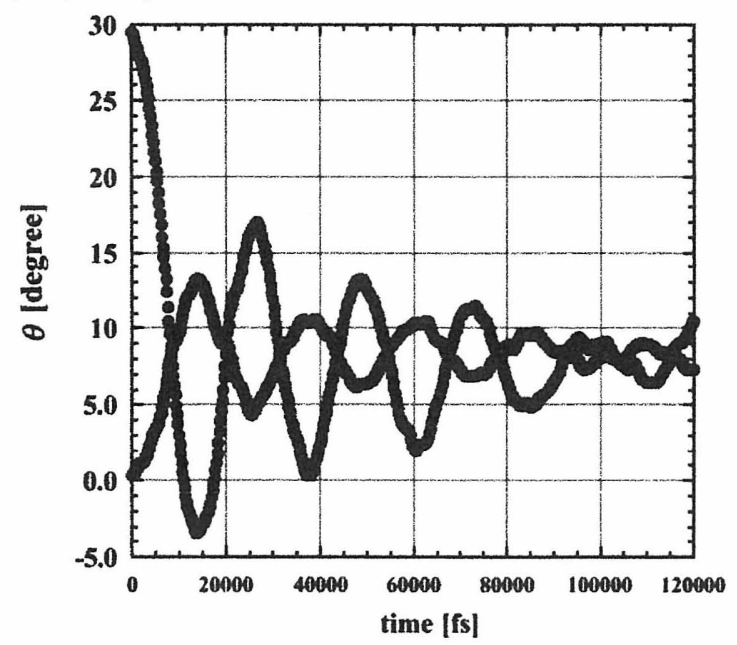

(b) (61+61) Chain Molecules

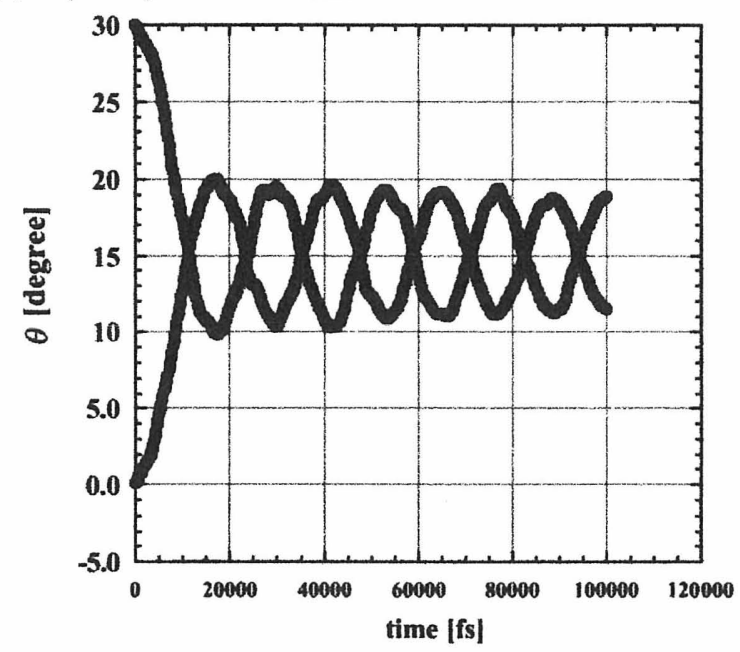

Fig. 2 Time evolution of angles for angle $\theta$, (a) $(61+29)$ chain-molecule system and (b) $(61+61)$ chain-molecule system.

References

1) S. Fujiwara and T. Sato, Phys. Rev. Lett. 80 (1998) 991.

2) S. Fujiwara and T. Sato, Molecular Simulation 21 (1999) 271.

3) S. Fujiwara and T. Sato, J. Chem. Phys. 1108 (1999) 9757.

4) H. Nakamura, S. Fujiwara and T. Sato, J. Phys. Soc. Jpn. 70 (2001) 943. 